\title{
Multi-Label Simple Points Definition for 3D Images Digital Deformable Model ${ }^{\star}$
}

\author{
Alexandre Dupas ${ }^{1}$, Guillaume Damiand ${ }^{2}$, and Jacques-Olivier Lachaud ${ }^{3}$ \\ 1 Université de Poitiers, CNRS, SIC-XLIM, UMR6172, F-86962, France \\ dupas@sic.univ-poitiers.fr \\ 2 Université de Lyon, CNRS, LIRIS, UMR5205, F-69622, France \\ guillaume.damiand@liris.cnrs.fr \\ 3 Université de Savoie, CNRS, LAMA, UMR5127, F-73376, France \\ jacques-olivier.lachaud@univ-savoie.fr
}

\begin{abstract}
The main contribution of this paper is the definition of multilabel simple points that ensures that the partition topology remains invariant during a deformable partition process. The definition is based on simple intervoxel properties and is easy to implement. A deformation process is carried out with a greedy energy minimization algorithm. A discrete area estimator is used to approach at best standard regularizers classically used in continuous energy minimizing methods. The effectiveness of our approach is shown on several 3D image segmentations.
\end{abstract}

Keywords: Simple Point, Deformable Model, Multi-Label Image.

\section{Introduction}

Segmentation is a crucial step in any image analysis process. Over the past twenty years, energy-minimizing techniques have shown a great potential for segmentation. They combine in a single framework two terms, one expressing the fit to data, the other describing shape priors and acting as a regularizer. Furthermore, as noted by many authors, the parameter balancing the two terms acts as a scale factor, providing a very natural multiscale analysis of images. Deformable models [14, Mumford-Shah approximation [19], geometric or geodesic active contours and other levelset variants 518624, are classical variational formulation (i.e. continuous) of such techniques. Our objective is to propose a novel energy-minimizing model for segmenting 3D images into regions, a kind of deformable digital partition with the following specific features.

(i) It is a purely digital formulation of energy minimization, which can be solved by combinatorial algorithms. We use a simple greedy algorithm.

(ii) The standard area regularizer is mimicked in this digital setting by a discrete geometric estimator.

(iii) It encodes both region structures and the geometry of their interfaces. It may thus incorporate any kind of fit to data energy, region-based like quadratic deviation [197] or contour-based like strong gradients [14].

\footnotetext{
^ Partially supported by the ANR program ANR-06-MDCA-008/FOGRIMMI.
} 
(iv) We propose a new method in order to guarantee that the topology of the whole partition is preserved during the deformation process.

Point (i) is interesting from a fundamental point of view. Continuous variational problems induce partial differential equations which are solved iteratively. They are most often bound to get stuck in local minimas, except in specific cases 971. To our knowledge, none of them are able to find the optimal image partition if more than two regions are expected. In discrete settings, the optimal solution to the two label partitioning is computable [11. For more regions, optimization algorithms can guarantee to be no further away than two times the optimal value 4, and scale-sets within pyramids present solutions that are experimentally very close to the optimal solution [12 21]. However, the regularization/shape prior term of these discrete methods is most often reduced to the number of surfels of the region boundaries, a very poor area estimator.

Point (ii) addresses this problem. We indeed propose an original regularization term which uses a discrete geometric estimator for computing the area of each surfel. Its principle is to extract maximal digital straight segments to estimate the surfel normal, area being a byproduct [17]. We get therefore a digital equivalent of continuous active surfaces minimizing their area, which is also an 3D extension of discrete deformable boundaries [16].

Point (iii) is important to get a versatile segmentation tool. According to the image characteristics, it is well known that contour or region based approaches are more or less adapted. From a minimization point of view, region-based energies are generally more "convex", thus easier to optimize [7/24. Our partition model allows to mix energies defined on regions and energies defined on boundaries. To our knowledge, very few explicit or implicit variational or deformable models can do that in 3D, except perhaps the work of Pons and Boissonnat [20], but they may not model energies depending on the inclusion between regions.

In this paper we focus on the last point which is mandatory for such deformable model. Point (iv) is important in several specific image applications where the topology of anatomic components is a prior information, like atlas matching. This is even truer in 3D images, where anatomic components are intertwined in a deterministic way. Preserving the topology of a two label partition in a discrete setting is generally done by computing and locating simple points 3 . Similar tools are used in level set techniques to control topology changes 1322 . For a multi-label partition, a few authors have proposed an equivalent to simple points in a discrete setting [232]. However, they are computationally too costly to be used to drive the evolution of a digital partition. We propose a new definition of simple points in multi-label partitions, that we call $M L$-Simple points (ML for Multi-Label). ML-Simpleness is stronger than simpleness, therefore deforming ML-Simple points preserves the partition topology. ML-Simpleness is easy to decide thanks to our intervoxel encoding. ML-Simpleness is sometimes a bit too restrictive and may forbid valid evolution. But our experiments show that it was not a problem in our context.

The paper is organized as follows. Section 2 recalls standard notions of digital geometry used later on. Section 3 presents the definition of ML-simpleness 
and proves that it implies simpleness. The ML-simpleness test derives from its definition. Section 4 describes a preliminary digital deformable partition model that uses ML-Simple points to ensure that the topology is preserved and Sect. 5. shows some first experiments.

\section{Preliminaries Notions}

A voxel is an element of the discrete space $\mathbb{Z}^{3}$. In the following, the symbol $I$ designates a $3 D$ image, which is a couple $\left(I_{d}, I_{f}\right)$, where $I_{d}$ is a set of voxels (the image domain) and $I_{f}$ is a map from $I_{d}$ to a set of colors or to a set of grey levels (the image values). Each voxel $v$ is associated with a label $l(v)$, a value in a given finite set $L$.

We use the classical notion of $\alpha$-adjacency, with $\alpha \in\{6,18,26\} . N_{\alpha}^{*}(v)$ is the set of voxels $\alpha$-adjacent to $v$, and $N_{\alpha}(v)=N_{\alpha}^{*}(v) \cup\{v\}$. An $\alpha$-path between two voxels $v_{1}$ and $v_{2}$ is a sequence of voxels from $v_{1}$ to $v_{2}$ such that each pair of consecutive voxels is $\alpha$-adjacent. A set of voxels $C$ is $\alpha$-connected iff there is an $\alpha$-path between any pair of voxels of $C$, with all its voxels in $C$.

The relation induced by being 6 -connected and having the same label is an equivalence relation over the image domain. The equivalence classes are the regions of the image. We consider an infinite region $r_{0}$ that surrounds the image (i.e. $r_{0}=\mathbb{Z}^{3} \backslash I_{d}$ ). The complement set of a region $X$ in $I_{d}$ is denoted by $\bar{X}$.

In order to describe the boundaries of the regions within an image, we use the classical notion of intervoxel [15. In the intervoxel framework, the discrete space is considered as a subdivision of the space in unit elements: voxels are unit cubes, surfels are unit squares between voxels, linels are unit segments between surfels, and pointels are the points between linels.

For a voxel $v$, we denote by $\operatorname{surfels}(v)$ the set of six surfels between $v$ and all its 6-neighbors. For a surfel $s$, we denote by linels $(s)$ the set of four linels between $s$ and its adjacent surfels, and for a linel $l$ we denote by pointels $(l)$ the set of two pointels between $l$ and its adjacent linels. We use also the notation $\operatorname{linels}(v)$ to denote the set of twelve linels around $v$. We say that a pointel $p$ and a linel $l$ (resp. a linel $l$ and a surfel $s$, a surfel $s$ and a voxel $v$ ) are incident if $p \in$ pointels $(l)($ resp. $l \in \operatorname{linels}(s), s \in \operatorname{surfels}(v))$.

We denote by $S F$ the set of boundary surfels of $I_{d}$, i.e. $S F=\{$ surfel $s \mid s$ separates two voxels with different labels\}. Note that all the surfels incident to a voxel of the infinite region belong to $S F$ since the label of the infinite region is by convention distinct from any other label. Given a voxel $v, s f(v)=\operatorname{surfels}(v) \cap S F$ is the set of boundary surfels incident to the voxel $v$.

The degree of a linel $d(l)$ is the number of boundary surfels incident to $l$. Note that $d(l)$ is $0,2,3$ or 4 , but never 1 . We denote by $d(l, v)$ the degree of $l$ restricted to boundary surfels incident to $v(s f(v))$.

We recall now notations and definition from [3]. The set of $\alpha$-connected components of a set of voxels $X$ is called $C_{\alpha}(X)$. The geodesic neighborhood of $v$ in $X$ of order $k$ is the set $N_{\alpha}^{k}(v, X)$ defined recursively by: $N_{\alpha}^{1}(v, X)=N_{\alpha}^{*}(v, X) \cap X$, and $N_{\alpha}^{k}(v, X)=\bigcup\left\{N_{\alpha}(Y) \cap N_{26}^{*}(v) \cap X, Y \in N_{\alpha}^{k-1}(v, X)\right\}$. 
In other words, $N_{\alpha}^{k}(v, X)$ is the set of voxels $x$ belonging to $N_{26}^{*}(v) \cap X$ such that it exists an $\alpha$-path $\pi$ from $v$ to $x$ of length at most $k$, all the voxels of $\pi$ belonging to $N_{26}^{*}(v) \cap X$.

In this paper, we use only the couple of neighborhood $(6,18)(6$ for object and 18 for background). In this framework, we obtain the 6 -geodesic neighborhood $G_{6}(x, X)=N_{6}^{3}(x, X)$ and the 18-geodesic neighborhood $G_{18}(x, X)=N_{18}^{2}(x, X)$.

From these notations, Bertrand 3 defines the notion of simple points in a $(6,18)$-connectivity as given in Definition 1

\section{Definition 1 (Simple Points [3])}

$A$ voxel $v$ is simple for a set $X$ if $\# C_{6}\left[G_{6}(v, X)\right]=\# C_{18}\left[G_{18}(v, \bar{X})\right]=1$, where $\# C_{k}[Y]$ denotes the number of $k$-connected components of a set $Y$.

\section{Multi-Label Simple Points}

Our goal is to modify an image partition by preserving the topology of described objects. Given a voxel $v$ in some region $R$, we want to remove $v$ from $R$ by modifying the label of $v$. The simple point definition is the main tool to control topology change. However, (1) we deal with multi-label images and not binary images; (2) we want to preserve the topology of regions but also the relations between surfaces (surface inclusion or intersection).

For these two reasons, we cannot directly use simple points. To our knowledge, there is no variant of simple points that follows these two constraints. Thus, we propose a new variant of simple point in multi-label images. Our main idea is to preserve the linels incident to the considered voxel in order to avoid removal or creation of surface intersections.

\subsection{Definition of Multi-Label Simple Points}

Definition 2 gives the definition of multi-label simple points (called ML-Simple points) which are points preserving both the topology of regions and the surface relations:

Definition 2 (ML-Simple Points). A voxel $v$ is $M L$-Simple if:

1. $\forall l \in \operatorname{linels}(v), d(l) \in\{0,2\}$;

2. The body of $s f(v)$ is homeomorphic to a 2-disk;

3. $\forall l \in \operatorname{linels}(v), d(l, v)=0 \Rightarrow d(l)=0$.

Intuitively, the three conditions of Definition 2 allow:

1. to avoid cases of several regions around voxel $v$ : this condition avoids $d(l)>2$ which is the case when more than 2 regions touch linel $l$.

2. to preserve the topology of the surface: if the set of surfels incident to $v$ and separating two voxels with different labels is not homeomorphic to a disk, the removal of voxel $v$ from its region induces a topological modification on the surface (cases A, D, H and J in Fig. 1); 
3. to preserve the relations between surfaces: if a linel $l$ is such that $d(l, v)=0$ and $d(l)>0$, the removal of voxel $v$ from its region forces the surface to touch another surface. This creates a new contact between two surfaces that were previously not adjacent (in Fig. 1 linels such that $d(l, v)=0$ are drawn in bold; for these linels, $d(l)$ must be equal to 0$)$.

Figure 1 displays the possible cases depending on the number of surfels of $s f(v)$ (from 0 to 6 ) for configurations satisfying condition (1). All other cases may be obtained from these cases by rotations and symmetries. In Fig. 1 cases $A, D, H$ and $J$ show non ML-Simple points since condition $(2)$ is violated. In case $A, s f(v)$ is empty and thus is not homeomorphic to a disk; in case $D, s f(v)$ is composed of two distinct components; in case $H, s f(v)$ is homeomorphic to an annulus, and in case $J, s f(v)$ is homeomorphic to a sphere. Note that case $I$ displays an ML-Simple point because there is no linel such that $d(l, v)=0$ thus condition (3) holds. For all other cases, points are ML-Simple if $d(l)=0$ for each bold linel $l$.

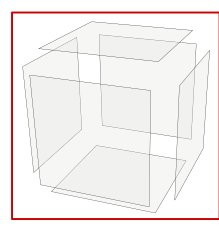

A

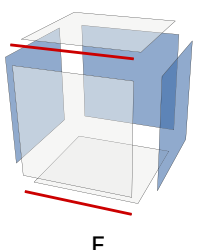

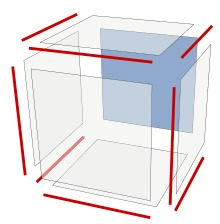

B

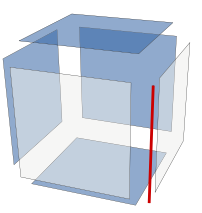

G

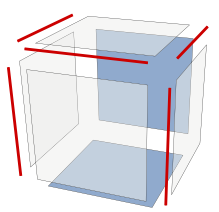

C

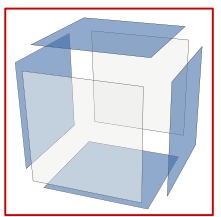

$\mathrm{H}$

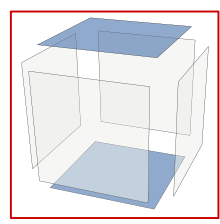

$\mathrm{D}$

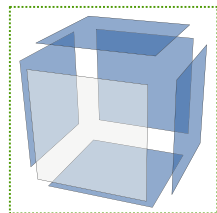

I

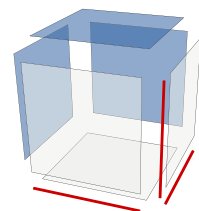

$\mathrm{E}$

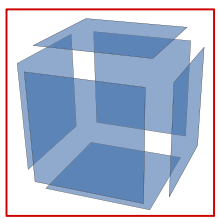

J

Fig. 1. All the possible cases depending on the number of surfels of $s f(v)$ (drawn in dark) incident to the considered voxel. All the configurations can be obtained from these 10 cases by rotation and symmetries. (A) Case of inner voxel with 0 boundary surfel. (B) 1 surfel. (C) and (D) 2 surfels. (E) and (F) 3 surfels. (G) and (H) 4 surfels. (I) 5 surfels. (J) Case of isolated voxel with 6 surfels. Bold linels are those with $d(l, v)=0$. Plain frames highlight non ML-Simple point configurations. The dashed frame highlights the case where the voxel is always ML-Simple.

\subsection{Multi-Label Simple Points Are Simple Points}

To prove that the topology of regions is preserved when removing a ML-Simple point, we show that ML-Simple points are simple points. Therefore, we prove that ML-Simple points occur only in binary neighborhood, which is required to prove that ML-Simple points are simple points since simple points are only defined for binary images.

Lemma 1. Let $v$ be an $M L$-Simple point, the number of distinct labels in $N_{18}(v)$ is two. 
Idea of the proof. The principle of the proof is to study the neighboorhod of $v$, and to show that all the voxels in $N_{18}(v)$ have either the same label as $v$, or have all the same label $w$, with $w \neq l(v)$. This is proved by contradiction, assuming this is not the case and then showing that each possible configuration contradicts one condition of Definition 2 .

Note that $v$ is an ML-Simple point does not imply that the number of distinct labels in $N_{26}(v)$ is two. However, Lemma 1 ensures that for each ML-Simple point $v$, removing $v$ from its region $R$ can be done in a unique way by setting $l(v)=w$ with $w$ the second label of $N_{18}(v)$. We naturally call this operation a swap, and denote it by $\operatorname{swap}(v)$, since $\operatorname{swap}(\operatorname{swap}(v))$ is the identity.

Now we show that the topologies of both the region $R$ containing $v$ and its complementary $\bar{R}$ are preserved by proving Proposition 1 which links ML-Simple points to simple points.

Proposition 1. If $v \in R$ is an $M L$-Simple point, then $v$ is a simple point for $R$.

Proof. We prove the contrapositive of Proposition 1, i.e. if $v$ is not a simple point for $R$, then $v$ is not an ML-Simple point. Let $n_{1}=\# C_{6}\left[G_{6}(v, R)\right]$ and $n_{2}=\# C_{18}\left[G_{18}(v, \bar{R})\right]$. Voxel $x$ is not simple in the four following cases: $(1)$ $n_{1}=0$, (2) $n_{2}=0$, (3) $n_{1} \geq 2$, (4) $n_{2} \geq 2$. We prove that, in each case, voxel $v$ is not an ML-Simple point.

1. $n_{1}=0$. There is no 6 -connected component of voxels belonging to $R$ in $G_{6}(v, R): v$ is an isolated point. In this case, $s f(v)$ contains all the surfels incident to $v$, and thus is homeomorphic to a sphere (case $\mathrm{J}$ in Fig. 1) which contradicts condition (2) of Definition 2 .

2. $n_{2}=0$. There is no 18 -connected component of voxels belonging to $\bar{R}$ in $G_{18}(v, \bar{R}): v$ is inside the region (i.e. all 18-neighbor voxels have the same label than $v$, case $\mathrm{A}$ in Fig. 1). In this case, $s f(v)$ is empty, which also contradicts condition (2).

3. $n_{1} \geq 2$ : there are at least two 6 -connected components of voxels belonging to $R$ in $G_{6}(v, R)$. If there are two 18-adjacent voxels $v_{1}$ and $v_{2}$ in two different connected components, then the voxel $v_{3} \neq v 6$-adjacent to $v_{1}$ and to $v_{2}$ belongs to $\bar{R}$ (otherwise there is only one connected component) and thus the linel $l$ incident to $v, v_{1}$ and $v_{2}$ is such that $d(l, v)=0$ (because the two voxels $v_{1}$ and $v_{2}$ belong to $R$ thus there is no surfel between these voxels and $v$ ) and $d(l)=2$ (there are two surfels, one between $v_{3}$ and $v_{1}$ and one between $v_{3}$ and $v_{2}$ ), which contradicts condition (3) of Definition 2 ,

If there is no two voxels $v_{1}$ and $v_{2}$ in two different connected components and which are 18-adjacent too, the connected components are separated by $v$ (case $\mathrm{H}$ in Fig. 1). In this case, $s f(v)$ is not homeomorphic to a disk (it is a annulus), which contradicts condition (2).

4. $n_{2} \geq 2$ : there are at least two 18 -connected components of voxels belonging to $\bar{R}$ in $G_{18}(v, \bar{R})$. If there are two voxels $v_{1}, v_{2} \in N_{6}(v)$ in two different connected components, then $v_{1}$ and $v_{2}$ are not 18 -adjacent (otherwise there 
is only one connected component). Hence the two surfels of $s f(v)$ between $v_{1}$ and $v$ and between $v_{2}$ and $v$ are not adjacent (case $\mathrm{D}$ in Fig. 1). Thus, $s f(v)$ is not homeomorphic to a disk, which contradicts condition (2) of Definition 2 .

If there is no two voxels of $N_{6}(v)$ in two different connected components, it means that one of them (say $v_{1}$ ) belongs to $N_{18}(v) \backslash N_{6}(v)$. The two 6neighbors of $v_{1}$ in $N_{6}(v)$ belong to $R$ (otherwise we are in the case of the previous paragraph). Hence the linel $l$ incident to $v_{1}$ and $v$ is such that $d(l, v)=0$ (because the two 6-neighbors of $v_{1}$ in $N_{6}(x)$ belong to $R$ thus there is no surfel between these surfels and $v)$. But there are two surfels between $v_{1}$ and its two 6-neighbors in $N_{6}(v)$ and $d(l)=2$. This contradicts condition (3).

Now we prove that the topology of the image is preserved (i.e. the topology of each region of the image is preserved and the surface relations are preserved). This proof is necessary since we deal with multiple regions and Proposition [1] proves only that the topology is preserved for binary cases.

Proposition 2. If $v$ is an $M L$-Simple point, the topology of the image partition is unchanged by swapping $v$.

Idea of the proof. The proof is made in two steps. First, we show that the topology of each region is preserved by using the link with simple points (Proposition 11). Second, we prove that the topology of surfaces is preserved (inclusion and adjacency between surfaces). With these two facts, we can prove that for each region, its Betti numbers 1 are preserved.

\subsection{Detection of Multi-Label Simple Points}

To efficiently retrieve intervoxel information, we use an intervoxel matrix to encode the borders of the regions in the 3D image. This matrix stores the state (on or off) of each intervoxel cell, determined by the three following rules:

- a surfel $s$ is on iff $s \in S F$ (i.e. $s$ is between 2 voxels with different labels);

- a linel $l$ is on iff $l$ is incident to $>2$ on surfels;

- a pointel $p$ is on iff $p$ is incident to 1 or $>2$ on linels.

Using this intervoxel matrix, Algo. 1] determines if voxel $v$ is ML-Simple. We prove that our algorithm returns true iff $v$ is an ML-Simple point.

Proof. For each linel $l$ incident to $v, l$ is on implies $d(l)>2$ which contradicts condition (1) of Definition 2, the algorithm returns false. The second test, if both surfels incident to $l$ and $v$ are off, corresponds to case $d(l, v)=0$. In this case, if at least one surfel incident to $l$ and not to $v$ is on, we have $d(l)>0$ which contradicts condition (1): the algorithm returns false. The last test verifies

${ }^{1}$ In $3 \mathrm{D}$, the first 3 Betti numbers count the number of connected components, the number of tunnels and the number of cavities. 


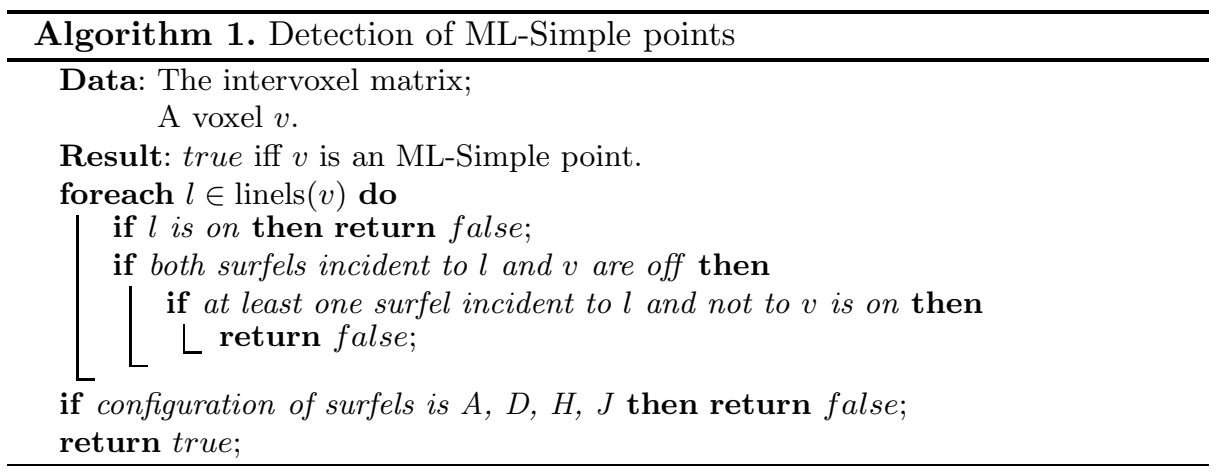

condition (2). We test if $s f(v)$ is homeomorphic to a disk by testing all the cases where this condition is not satisfied (cases A, D, H, J in Fig. 1) and returns false in such a case. After all these tests, the three conditions of Definition 2 are satisfied, thus $v$ is ML-Simple. The algorithm returns true accordingly.

The complexity of Algo. 1 is $O(1)$. There are 12 linels in linels $(v)$, and each test (if a cell is on or off) is an atomic operation. Checking if the configuration is A, $\mathrm{D}, \mathrm{H}$ or $\mathrm{J}$ is easily achieved in constant time for each case, just by probing some particular surfels.

\section{Deformable Model Process}

We define a digital deformable partition model, whose geometry is encoded with an intervoxel matrix. The elementary deformations are swaps of ML-Simple voxels. Proposition 2 ensures the preservation of the topology of the partition. The deformation is governed by an energy-minimizing process. The energy used here is a preliminary and simple version allowing to show the feasibility of a deformable partition model based on ML-Simple voxels swaps. The energy of a partition is the sum of the energies of each digital surface $S$ between pairs of regions $\left(r_{1}, r_{2}\right)$. The total energy for $S$ is defined as $E(S)=\omega_{r} E_{r}(S)+\omega_{s} E_{s}(S)$, where $E_{r}$ and $E_{s}$ are respectively the region and surface energies. Parameters $\omega_{r}$ and $\omega_{s}$ are the weights defining the relative significance of the corresponding terms.

Energy $E_{r}$ is an energy describing the quality of the fit of regions to image data: $E_{r}(S)=M S E\left(r_{1}\right)+M S E\left(r_{2}\right)$ where $M S E(x)$ is the Mean Squared Error of region $x . E_{r}(S)$ decreases when the region becomes more homogeneous.

Energy $E_{s}$ is based on a discrete area estimator proposed in [17. The area estimation is not the same whether it is computed on one side or the other of the surface. Thus, the energy of $S$ is defined as $E_{s}(S)=\sum_{s \in S} \operatorname{area}_{r_{1}}(s)+\operatorname{area}_{r_{2}}(s)$ where $\operatorname{area}_{r_{1}}(s)$ and $\operatorname{area}_{r_{2}}(s)$ are the area estimators for a surfel $s$ considering respectively the side of region $r_{1}$ and the side of region $r_{2} . E_{s}$ decreases when the surface becomes smoother. 
The principle of the deformation process of one surface $S$ is the following. The initial energy is computed. For each surfel $s$ of $S$, the process tries two swaps, one for each voxel incident to $s: v_{1}$ and $v_{2}$. If this voxel is an ML-Simple point we temporary swap it and compute the energy associated to the move. The move of minimum energy is selected. If its energy is lower than the initial energy, we apply the deformation and set the new initial energy. In the case of an energy minimization process, the deformation algorithm is executed on every border faces represented in the intervoxel matrix. The process iterates until no deformations occur (i.e. a local minimum energy is reached). Since we process surfels, and since a swap is made if and only if the global energy strictly decreases, deformations will stop at some point whatever the input configuration.

\section{Experiments}

In this section we present three experiments on the deformation process and the energies used in the paper.

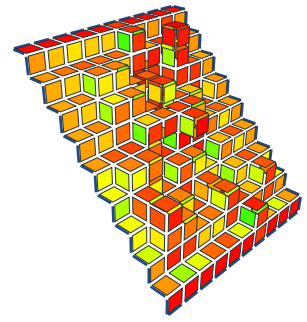

(a)

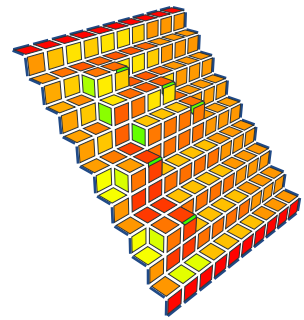

(b)

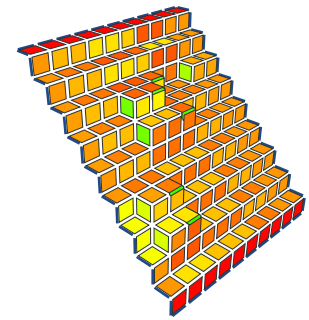

(c)

Fig. 2. (a) A noisy discrete plane surface. (b) Minimization of the number of surfels. (c) Minimization of the area based on the discrete estimator.

The first experiment highlights the interest of the discrete area estimator for regularization. We experiment a deformation process on a noisy plane surface in order to retrieve the optimal discrete plane. Two energies are compared: one using the number of surfels and one using the discrete area estimator. Figure 2a shows the noisy discrete area $\left(|S|=240, E_{s}(S)=338.0\right)$ corresponding to an inclined plane: the optimal result is $\left(|S|=180, E_{s}(S)=259.4\right)$. Figure $2 \mathrm{~b}$ is the result of the energy minimization process using the energy based on the number of surfels $\left(|S|=190, E_{s}(S)=266.0\right)$. Figure $2 \mathrm{c}$ shows the result of the energy minimization process using the energy based on the discrete area estimator $\left(|S|=192, E_{s}(S)=262.2\right)$. The discrete area estimator gives a better visual and estimated area than the energy based on the number of surfels. Remark that, in both cases, a local minimum is reached.

The second experiment shows a segmentation of a 3D medical image with a poor initialization, in a way similar to continuous deformable partition models 
24. Starting with a topologically correct segmentation of the image, the deformation process is used to retrieve shapes in the image while keeping topological information. The algorithm is applied on a simulated MRI brain image obtained from [8]. According to a-priori knowledge the image is composed of a sphere with two surrounding shells. Figure $3 \mathrm{a}$ shows a slice of the original image, the initial partition on the same slice is presented Fig. $3 \mathrm{~b}$ and the resulting segmentation is shown in Fig. 3c). The algorithm ensures that the topology of the last segmentation is the same as the topology of the image partitionned by three surfaces. The resulting partition is not satisfactory due to the expression of the energies which needs to be adressed in future works.

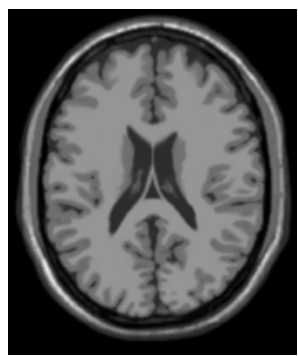

(a)

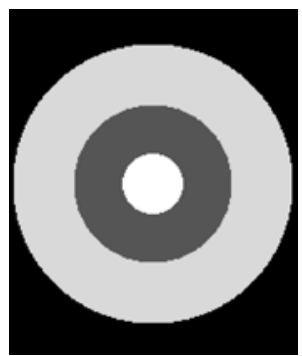

(b)

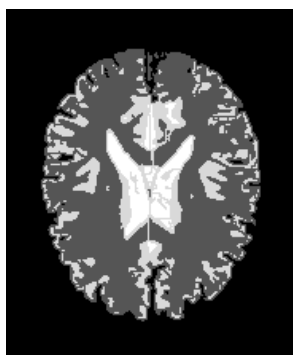

(c)

Fig. 3. (a) Slice of a simulated MRI brain image. (b) Initial partition with three overlapping spheres. (c) Resulting segmentation after deformation.

The last experiment consists in optimizing an initial segmentation containing several regions in order to enhance the result. The first partition, produced by a split and merge algorithm, contains block shaped regions. The deformation slightly modifies surfaces of the image to get a better partition according to the criterion. Figure $4 \mathrm{a}$ and Fig. $4 \mathrm{~b}$ present a slice of the partition before and after the deformation processes. Borders of regions match more accurately image data. Figure 4c and Fig. 4d present the surface of the central dark region region before and after the deformation processes.

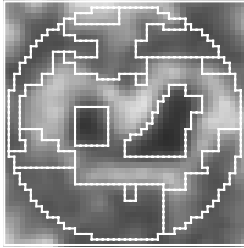

(a)

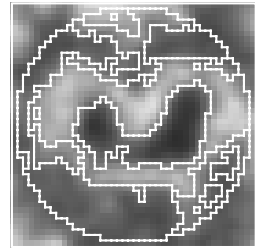

(b)

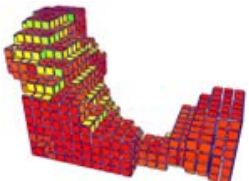

(c)

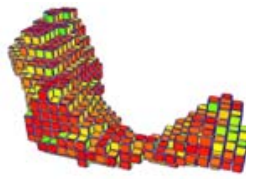

(d)

Fig. 4. (a) Slice of the initial segmentation. (b) Same slice after deformation. (c) Surface of the stripped region. (d) Same surface after deformation. 


\section{Conclusion}

The main contributions of this work are: (i) The definition of ML-Simple points: a voxel is ML-Simple if its removal preserves the topology of the partition. The ML-Simple test algorithm is local, short and easy to implement. (ii) Our method is generic: regions and surfaces information can be mixed to define energies specialized for various applications. (iii) Our work deals with arbitrary multilabel image partitions: we can deform any number of surfaces while preserving their topology. The overall computational complexity depends on the number of surfels of the partition, not on its topological complexity. These interests have been illustrated in several preliminaries experiments. We may either deform an initial set of arbitrary surfaces like the example of three included spheres that fit a brain image, or smooth an initial partition obtained from a preliminary segmentation.

In future works, we plan to extend the notion of ML-Simple point to allow deformation of surface intersections. Currently, if there is an intersection between two surfaces, we leave unchanged both the topology and the geometry of this intersection since linels are preserved. We think that the definition can be extended to preserve the topology while allowing intersections to be moved. Another prospect is to improve the energies used in the deformable model to have a better fit with the image data. The discrete area estimator could also be improved, first by making it linear-time in the same way as the $2 \mathrm{D}$ case, secondly by making it dynamic to avoid global recomputation. This would allow the processing of big 3D images. Another research track is to find an area estimator with less local minima, in a way similar to [10] in 2D.

\section{References}

1. Ardon, R., Cohen, L.D.: Fast constrained surface extraction by minimal paths. International Journal on Computer Vision 69(1), 127-136 (2006)

2. Bazin, P.-L., Ellingsen, L.M., Pham, D.L.: Digital homeomorphisms in deformable registration. In: Karssemeijer, N., Lelieveldt, B. (eds.) IPMI 2007. LNCS, vol. 4584, pp. 211-222. Springer, Heidelberg (2007)

3. Bertrand, G.: Simple points, topological numbers and geodesic neighborhoods in cubic grids. Pattern Recognition Letters 15(10), 1003-1011 (1994)

4. Boykov, Y., Veksler, O., Zabih, R.: Fast approximate energy minimization via graph cuts. IEEE Transactions on Pattern Analysis and Machine Intelligence 23(11), 1222-1239 (2001)

5. Caselles, V., Catte, F., Coll, T., Dibos, F.: A geometric model for active contours. Numerische Mathematik 66, 1-31 (1993)

6. Caselles, V., Kimmel, R., Sapiro, G., Sbert, C.: Minimal surfaces based object segmentation. IEEE Trans. Pattern Anal. Mach. Intell. 19(4), 394-398 (1997)

7. Chan, T.F., Vese, L.A.: Active contours without edges. IEEE Trans. on Image Processing 10(2), 266-277 (2001)

8. Cocosco, C.A., Kollokian, V., Kwan, R.K.-S., Evans, A.C.: Brainweb: Online interface to a 3d MRI simulated brain database. In: Proc. of 3-rd Int. Conference on Functional Mapping of the Human Brain, Copenhagen, Denmark (May 1997) 
9. Cohen, L.D., Kimmel, R.: Global minimum for active contour models: a minimal path approach. Int. Journal of Computer Vision 24(1), 57-78 (1997)

10. de Vieilleville, F., Lachaud, J.-O.: Toward a digital deformable model simulating open active contours. In: Brlek, S., Reutenauer, C., Provençal, X. (eds.) DGCI 2009. LNCS, vol. 5810, pp. 156-167. Springer, Heidelberg (2009)

11. Greig, D., Porteous, B., Seheult, A.: Exact maximum a posteriori estimation for binary images. Journal of the Royal Statistical Society (B) 51(2), 271-279 (1989)

12. Guigues, L., Cocquerez, J.-P., Le Men, H.: Scale-sets image analysis. International Journal on Computer Vision 68(3), 289-317 (2006)

13. Han, X., Xu, C., Prince, J.L.: A topology preserving level set method for geometric deformable models. IEEE Trans. on Pattern Analysis and Machine Intelligence 25(6), 755-768 (2003)

14. Kass, M., Witkin, A., Terzopoulos, D.: Snakes: Active contour models. International Journal of Computer Vision 1(4), 321-331 (1988)

15. Kovalevsky, V.A.: Finite topology as applied to image analysis 46, 141-161 (1989)

16. Lachaud, J.-O., Vialard, A.: Discrete deformable boundaries for the segmentation of multidimensional images. In: Arcelli, C., Cordella, L.P., Sanniti di Baja, G. (eds.) IWVF 2001. LNCS, vol. 2059, pp. 542-551. Springer, Heidelberg (2001)

17. Lachaud, J.-O., Vialard, A.: Geometric measures on arbitrary dimensional digital surfaces. In: Nyström, I., Sanniti di Baja, G., Svensson, S. (eds.) DGCI 2003. LNCS, vol. 2886, pp. 434-443. Springer, Heidelberg (2003)

18. Malladi, R., Sethian, J.A., Vemuri, B.C.: Shape Modelling with Front Propagation: A Level Set Approach. IEEE Trans. on Pattern Analysis and Machine Intelligence 17(2), 158-174 (1995)

19. Mumford, D., Shah, J.: Optimal approximations by piecewise smooth functions and associated variational problems. Comm. Pure Appl. Math. 42, 577-684 (1989)

20. Pons, J.-P., Boissonnat, J.-D.: Delaunay deformable models: Topology-adaptive meshes based on the restricted Delaunay triangulation. In: Proc. IEEE Conference on Computer Vision and Pattern Recognition, pp. 1-8 (2007)

21. Pruvot, J.H., Brun, L.: Scale set representation for image segmentation. In: Escolano, F., Vento, M. (eds.) GbRPR. LNCS, vol. 4538, pp. 126-137. Springer, Heidelberg (2007)

22. Ségonne, F.: Active contours under topology control - genus preserving level sets. Int. Journal of Computer Vision 79, 107-117 (2008)

23. Ségonne, F., Pons, J.-P., Grimson, W.E.L., Fischl, B.: Active contours under topology control genus preserving level sets. In: Int. Workshop Computer Vision for Biomedical Image Applications, pp. 135-145 (2005)

24. Vese, L.A., Chan, T.F.: A multiphase level set framework for image segmentation using the Mumford and Shah model. Int. J. Comput. Vis. 50(3), 271-293 (2002) 